\title{
Unique structure is found in human chromosome DNA sequences
}

\section{Zhiyu Chen}

Peiyou Education School, Changlin Road 959\# 106 107 room, Shanghai, China

\section{Email address:}

Seeker@stu.cpu.edu.cn

\begin{abstract}
We have found that each human chromosome has an unique structure, which is about 1 percent of the length of the entire chromosome. We analyze the NCBI all other 117 kinds different creatures, we analyze their every chromosome sequence, in these 3500 chromosome sequences, there has not a chromosome which has the same unique structure as the human chromosomes, we think the unique structure only exists in human chromosomes and does not exist in other creatures chromosomes. We also find this structure position is very near the centromere in every human chromosome. we give our report to the world, we hope other researchers do more research deeply, it is very important to human.
\end{abstract}

Keywords: ATGC/CGTA, unique structure, human chromosome, centromere

\section{Introduction}

The Human Genome Project (HGP) was an international scientific research project with the goal of determining the sequence of nucleotide base pairs that make up human DNA, and of identifying and mapping all of the genes of the human genome from both a physical and a functional standpoint.[1] It remains the world's largest collaborative biological project.[2] After the idea was picked up in 1984 by the US government when the planning started, the project formally launched in 1990 and was declared complete in 2001[3]. Funding came from the US government through the National Institutes of Health (NIH) as well as numerous other groups from around the world. A parallel project was conducted outside government by the Celera Corporation, or Celera Genomics, which was formally launched in 1998. Most of the government-sponsored sequencing was performed in twenty universities and research centers in the United States, the United Kingdom, Japan, France, Germany, Spain and China.[4]

DNA sequencing is the process of determining the precise order of nucleotides within a DNA molecule. It includes any method or technology that is used to determine the order of the four bases - adenine, guanine, cytosine, and thymine - in a strand of DNA. The advent of rapid DNA sequencing methods has greatly accelerated biological and medical research and discovery.[5]

Knowledge of DNA sequences has become indispensable for basic biological research, and in numerous applied fields such as medical diagnosis, biotechnology, forensic biology, virology and biological systematics. The rapid speed of sequencing attained with modern DNA sequencing technology has been instrumental in the sequencing of complete DNA sequences, or genomes of numerous types and species of life, including the human genome and other complete DNA sequences of many animal, plant, and microbial species.
The first DNA sequences were obtained in the early 1970s by academic researchers using laborious methods based on two-dimensional chromatography. Following the development of fluorescence-based sequencing methods with a DNA sequencer,[6] DNA sequencing has become easier and orders of magnitude faster.[7]

Sequencing of full genomes

The first full DNA genome to be sequenced was that of bacteriophage $\varphi$ X174 in 1977.[8] Medical Research Council scientists deciphered the complete DNA sequence of the Epstein-Barr virus in 1984, finding it contained 172,282 nucleotides. Completion of the sequence marked a significant turning point in DNA sequencing because it was achieved with no prior genetic profile knowledge of the virus.[9]

A non-radioactive method for transferring the DNA molecules of sequencing reaction mixtures onto an immobilizing matrix during electrophoresis was developed by Pohl and co-workers in the early 1980s.[10][11] Followed by the commercialization of the DNA sequencer "DirectBlotting-Electrophoresis-System GATC 1500" by GATC Biotech, which was intensively used in the framework of the EU genome-sequencing programme, the complete DNA sequence of the yeast Saccharomyces cerevisiae chromosome II.[12] Leroy E. Hood's laboratory at the California Institute of Technology announced the first semi-automated DNA sequencing machine in 1986.[13] This was followed by Applied Biosystems' marketing of the first fully automated sequencing machine, the ABI 370, in 1987 and by Dupont's Genesis 2000[14] which used a novel fluorescent labeling technique enabling all four dideoxynucleotides to be identified in a single lane. By 1990, the U.S. National Institutes of Health (NIH) had begun large-scale sequencing trials on Mycoplasma capricolum, Escherichia coli, Caenorhabditis elegans, and Saccharomyces cerevisiae at a cost of US\$0.75 per base. Meanwhile, sequencing of human cDNA sequences called expressed sequence tags began in Craig Venter's lab, an attempt to capture the coding fraction 
of the human genome.[15] In 1995, Venter, Hamilton Smith, and colleagues at The Institute for Genomic Research (TIGR) published the first complete genome of a free-living organism, the bacterium Haemophilus influenzae. The circular chromosome contains $1,830,137$ bases and its publication in the journal Science[16] marked the first published use of whole-genome shotgun sequencing, eliminating the need for initial mapping efforts.

By 2001, shotgun sequencing methods had been used to produce a draft sequence of the human genome.[17][18]

\section{Maxam-Gilbert sequencing}

Allan Maxam and Walter Gilbert published a DNA sequencing method in 1977 based on chemical modification of DNA and subsequent cleavage at specific bases.[19] Also known as chemical sequencing, this method allowed purified samples of double-stranded DNA to be used without further cloning. This method's use of radioactive labeling and its technical complexity discouraged extensive use after refinements in the Sanger methods had been made.

Maxam-Gilbert sequencing requires radioactive labeling at one 5' end of the DNA and purification of the DNA fragment to be sequenced. Chemical treatment then generates breaks at a small proportion of one or two of the four nucleotide bases in each of four reactions $(\mathrm{G}, \mathrm{A}+\mathrm{G}, \mathrm{C}, \mathrm{C}+\mathrm{T})$. The concentration of the modifying chemicals is controlled to introduce on average one modification per DNA molecule. Thus a series of labeled fragments is generated, from the radiolabeled end to the first "cut" site in each molecule. The fragments in the four reactions are electrophoresed side by side in denaturing acrylamide gels for size separation. To visualize the fragments, the gel is exposed to X-ray film for autoradiography, yielding a series of dark bands each corresponding to a radiolabeled DNA fragment, from which the sequence may be inferred.[19]

\section{Chain-termination methods}

The chain-termination method developed by Frederick Sanger and coworkers in 1977 soon became the method of choice, owing to its relative ease and reliability.[20][21] When invented, the chain-terminator method used fewer toxic chemicals and lower amounts of radioactivity than the Maxam and Gilbert method. Because of its comparative ease, the Sanger method was soon automated and was the method used in the first generation of DNA sequencers.

Sanger sequencing is the method which prevailed from the 1980s until the mid-2000s. Over that period, great advances were made in the technique, such as fluorescent labelling, capillary electrophoresis, and general automation. These developments allowed much more efficient sequencing, leading to lower costs. The Sanger method, in mass production form, is the technology which produced the first human genome in 2001, ushering in the age of genomics. However, later in the decade, radically different approaches reached the market, bringing the cost per genome down from $\$ 100$ million in 2001 to $\$ 10,000$ in 2011.[22]

Shotgun sequencing

Shotgun sequencing is a sequencing method designed for analysis of DNA sequences longer than 1000 base pairs, up to and including entire chromosomes. This method requires the target DNA to be broken into random fragments. After sequencing individual fragments, the sequences can be reassembled on the basis of their overlapping regions.[24]

Bridge PCR

Another method for in vitro clonal amplification is bridge PCR, in which fragments are amplified upon primers attached to a solid surface[23][25][26] and form "DNA colonies" or "DNA clusters". This method is used in the Illumina Genome Analyzer sequencers. Single-molecule methods, such as that developed by Stephen Quake's laboratory (later commercialized by Helicos) are an exception: they use bright fluorophores and laser excitation to detect base addition events from individual DNA molecules fixed to a surface, eliminating the need for molecular amplification.[27]

The centromere is the specialized DNA sequence of a chromosome that links a pair of sister chromatids (a dyad).[28] During mitosis, spindle fibers attach to the centromere via the kinetochore.[29] Centromeres were first thought to be genetic loci that direct the behavior of chromosomes.

The physical role of the centromere is to act as the site of assembly of the kinetochores - a highly complex multiprotein structure that is responsible for the actual events of chromosome segregation - i.e. binding microtubules and signalling to the cell cycle machinery when all chromosomes have adopted correct attachments to the spindle, so that it is safe for cell division to proceed to completion and for cells to enter anaphase.[30]

There are, broadly speaking, two types of centromeres. "Point centromeres" bind to specific proteins that recognize particular DNA sequences with high efficiency.[31] Any piece of DNA with the point centromere DNA sequence on it will typically form a centromere if present in the appropriate species. The best characterised point centromeres are those of the budding yeast, Saccharomyces cerevisiae. "Regional centromeres" is the term coined to describe most centromeres, which typically form on regions of preferred DNA sequence, but which can form on other DNA sequences as well.[31] The signal for formation of a regional centromere appears to be epigenetic. Most organisms, ranging from the fission yeast Schizosaccharomyces pombe to humans, have regional centromeres.

Regarding mitotic chromosome structure, centromeres represent a constricted region of the chromosome (often referred to as the primary constriction) where two identical sister chromatids are most closely in contact. When cells enter mitosis, the sister chromatids (the two copies of each chromosomal DNA molecule resulting from DNA replication in chromatin form) are linked along their length by the action of the cohesin complex. It is now believed that this complex is mostly released from chromosome arms during prophase, so that by the time the chromosomes line up at the mid-plane of the mitotic spindle (also known as the metaphase plate), the last place where they are linked with one another is in the chromatin in and around the centromere.[32] 


\section{Method}

See figure1, long chromosome sequence is segmented into shot-length segments, every segment have same length $10000 \mathrm{bp}$.

To every sample segment, we calculate its complementary sequence, in sample sequence and its complementary sequence, we both calculate their oligonucleotide ATGC number and oligonucleotide CGTA number, then calculate the ratio of this segment sequence.

Suppose : In sample segment, $\mathrm{n} 1$ is oligonucleotide ATGC number, $\mathrm{n} 2$ is oligonucleotide CGTA number, in its complementary sequence, $\mathrm{n} 3$ is oligonucleotide ATGC number, $\mathrm{n} 4$ is oligonucleotide CGTA number

$$
\text { Ratio }=(\mathbf{n} 1 / \mathbf{n} 2) /(\mathbf{n} 3 / \mathbf{n} 4)
$$

\section{the whole DNA sequence}

segment by filespliter.exe

segment $1(10 \mathrm{Kbp}) \quad$ segment $2(10 \mathrm{Kbp}) \quad$ segment $3(10 \mathrm{Kbp}) \quad$ segment ... $\quad$ segment $\mathbf{n}(10 \mathrm{Kbp})$

X-axis:

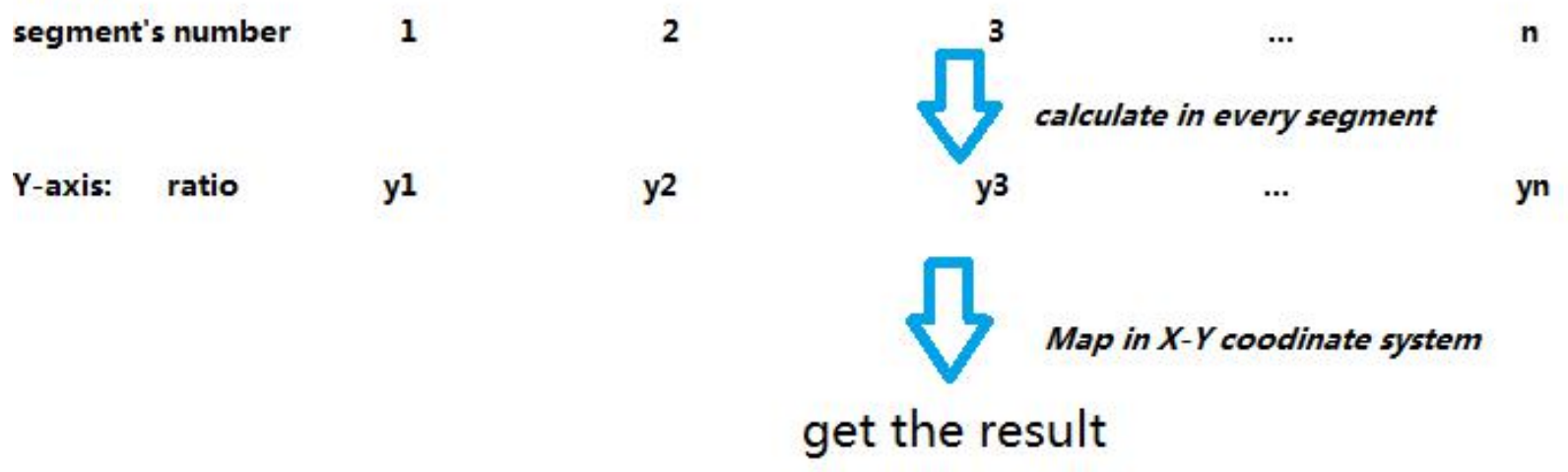

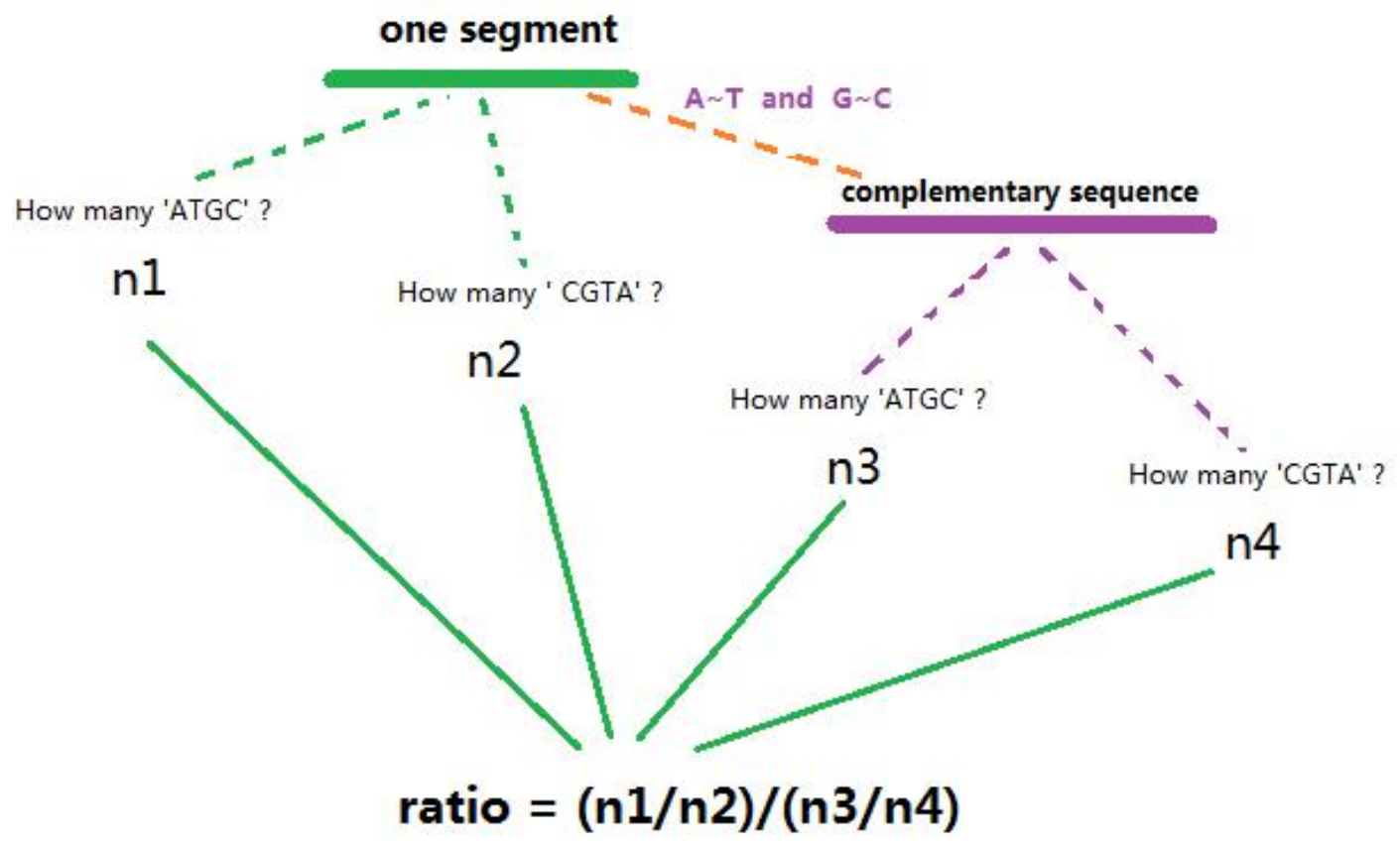

Figure 1. method. 
Here give all Matlab analyze program: the example file ' hs_ref_GRCh38.p12_chr2.fa' is downloaded from NCBI

fid = fopen('hs_ref_GRCh38.p12_chr2.fa'); \% to other DNA sequence, please use filename to replace the filename here

fseek(fid, 0, 'eof');

fsize $=$ ftell(fid);

$\mathrm{q} 123=\mathrm{fix}(\mathrm{fsize} / 10000)$;

test $=$ zeros $(1, \mathrm{q} 123)$;

$\mathrm{ff}=\mathrm{fclose}(\mathrm{fid})$;

fid = fopen('hs_ref_GRCh38.p12_chr2.fa'); \% to other DNA

sequence, please use filename to replace the filename here

for $\mathrm{qw}=1: \mathrm{q} 123$

$\mathrm{aa}=$ fread(fid,10000);

$\mathrm{a}=\mathrm{aa}$;

$\mathrm{qq}=10000$

$1=\mathrm{a} * 0$;

num $1=0$;

for $i=1: q q$

if a(i) $>64$

num $1=$ num $1+1$;

$1($ num1 $)=a(i)$;

else

end

end

$\mathrm{c}=1(1$ :num1);

clear a

clear $b$

$\mathrm{a}=\mathrm{c} * 0$;

num $2=0$;

for $\mathrm{i}=1$ :num 1

if $\left(\mathrm{c}(\mathrm{i})-\mathrm{A}^{\prime}\right) *\left(\mathrm{c}(\mathrm{i})-\mathrm{a}^{\prime}\right)==0$

num $2=$ num $2+1$;

$\mathrm{a}($ num 2$)=\mathrm{i}$;

else

end

end

$\mathrm{b}=\mathrm{c}^{*} 0$;

atgcip $=0$;

for $\mathrm{i}=1$ :num $2-3$

temp $=\mathrm{abs}\left(\mathrm{c}(\mathrm{a}(\mathrm{i})+1)-\mathrm{t}^{\prime}\right) * \mathrm{abs}\left(\mathrm{c}(\mathrm{a}(\mathrm{i})+1)-\mathrm{T}^{\prime}\right)+\mathrm{abs}(\mathrm{c}(\mathrm{a}(\mathrm{i})+2)-$

'g')*abs(c(a(i)+2)-'G')+abs(c(a(i)+3)-'c')*abs(c(a(i)+3)-'C');

if temp $=0$

$\mathrm{b}(\mathrm{a}(\mathrm{i}))=1$;

atgcip $=$ atgcip +1 ;

else

end

end

clear a

clear $b$

$\mathrm{a}=\mathrm{c} * 0$;

num $2=0$;

for $i=1$ :num 1

if $\left(\mathrm{c}(\mathrm{i})-\mathrm{C}^{\prime}\right) *\left(\mathrm{c}(\mathrm{i})-\mathrm{c}^{\prime}\right)==0$

num $2=$ num $2+1$

$\mathrm{a}($ num 2$)=\mathrm{i}$;

else

end

end $\mathrm{b}=\mathrm{c} * 0$

cgtaip $=0$;

for $\mathrm{i}=1$ :num $2-3$

temp $=$ abs $\left(\mathrm{c}(\mathrm{a}(\mathrm{i})+1)-\mathrm{g}^{\prime}\right) * \mathrm{abs}\left(\mathrm{c}(\mathrm{a}(\mathrm{i})+1)-\mathrm{G}^{\prime}\right)+\mathrm{abs}(\mathrm{c}(\mathrm{a}(\mathrm{i})+2)-$

't')*abs(c(a(i)+2)-'T')+abs(c(a(i)+3)-'a')*abs(c(a(i)+3)-'A');

if temp $=0$

$b(a(i))=1$;

cgtaip $=$ cgtaip +1 ;

else

end

end

clear a

clear b

$\mathrm{a}=\mathrm{c}^{*} 0$;

num $2=0$;

for $i=1$ :num 1

if $\left(\mathrm{c}(\mathrm{i})-^{\prime} \mathrm{G}^{\prime}\right) *\left(\mathrm{c}(\mathrm{i})-\mathrm{g}^{\prime}\right)==0$

num $2=$ num $2+1$;

$\mathrm{a}($ num 2$)=\mathrm{i}$;

else

end

end

$\mathrm{b}=\mathrm{c} * 0$

gcatip $=0$;

for $i=1$ :num $2-3$

temp $=\mathrm{abs}\left(\mathrm{c}(\mathrm{a}(\mathrm{i})+1)-\mathrm{c}^{\prime}\right) * \mathrm{abs}\left(\mathrm{c}(\mathrm{a}(\mathrm{i})+1)-{ }^{\prime} \mathrm{C}^{\prime}\right)+\mathrm{abs}(\mathrm{c}(\mathrm{a}(\mathrm{i})+2)-$

'a')*abs(c(a(i)+2)-'A')+abs(c(a(i)+3)-''t')*abs(c(a(i)+3)-'T');

if temp $==0$

$b(a(i))=1$;

gcatip $=$ gcatip +1 ;

else

end

end

clear a

clear b

$\mathrm{a}=\mathrm{c}^{*} 0$;

num $2=0$;

for $i=1$ :num 1

if $\left(\mathrm{c}(\mathrm{i})-\mathrm{T}^{\prime}\right) *\left(\mathrm{c}(\mathrm{i})-\mathrm{t}^{\prime}\right)==0$

num $2=$ num $2+1$;

$\mathrm{a}($ num 2$)=\mathrm{i}$;

else

end

end

$\mathrm{b}=\mathrm{c} * 0$;

tacgip $=0$;

for $\mathrm{i}=1$ :num $2-3$

temp $=a b s\left(c(a(i)+1)-a^{\prime}\right) * a b s\left(c(a(i)+1)-A^{\prime}\right)+a b s(c(a(i)+2)-$

'c')*abs(c(a(i)+2)-'C')+abs(c(a(i)+3)-'g')*abs(c(a(i)+3)-'G');

if temp $==0$

$\mathrm{b}(\mathrm{a}(\mathrm{i}))=1$;

tacgip=tacgip +1 ;

else

end

end

test(qw)=atgcip/cgtaip $*$ tacgip/gcatip;

clear a

end

$\mathrm{ff}=\mathrm{fclose}(\mathrm{fid})$;

plot(test) 


\section{Results}
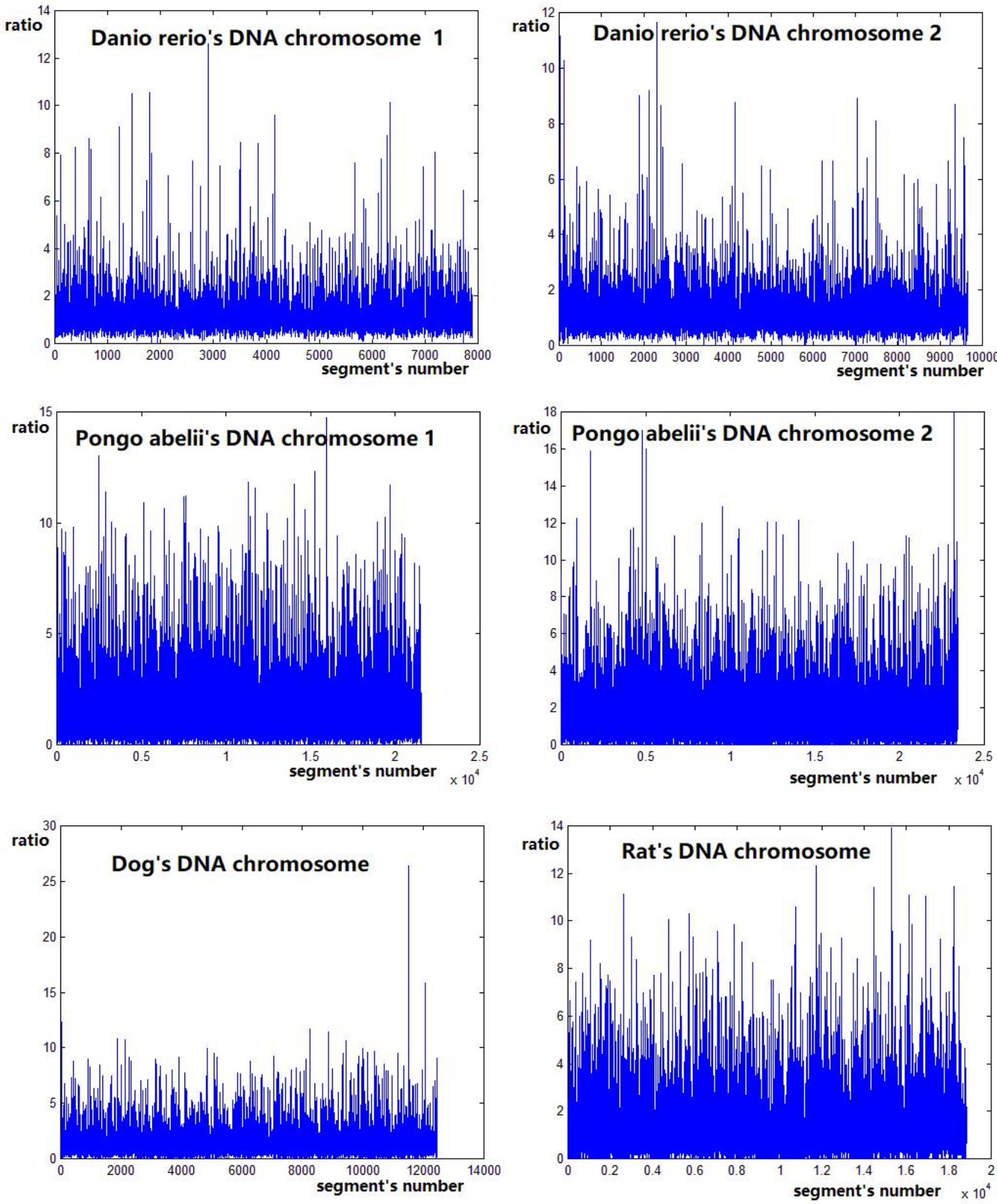

Figure 2. examples chromosome map. 
These examples chromosomes are all downloaded from ftp.ncbi.nih.gov/genomes/, to every chromosome sequence, first put the sequence file into matlab work space,then use the filename to replace the filename hs_ref_GRCh38.p12_chr2.fa ' in the example matlab program, run the program, you can get the result.
In figure 2, we choose danio rerio, pongo, dog and rat, we see that different creatures have similar maps.

Now, we test human chromosome, all datas are from NCBI 

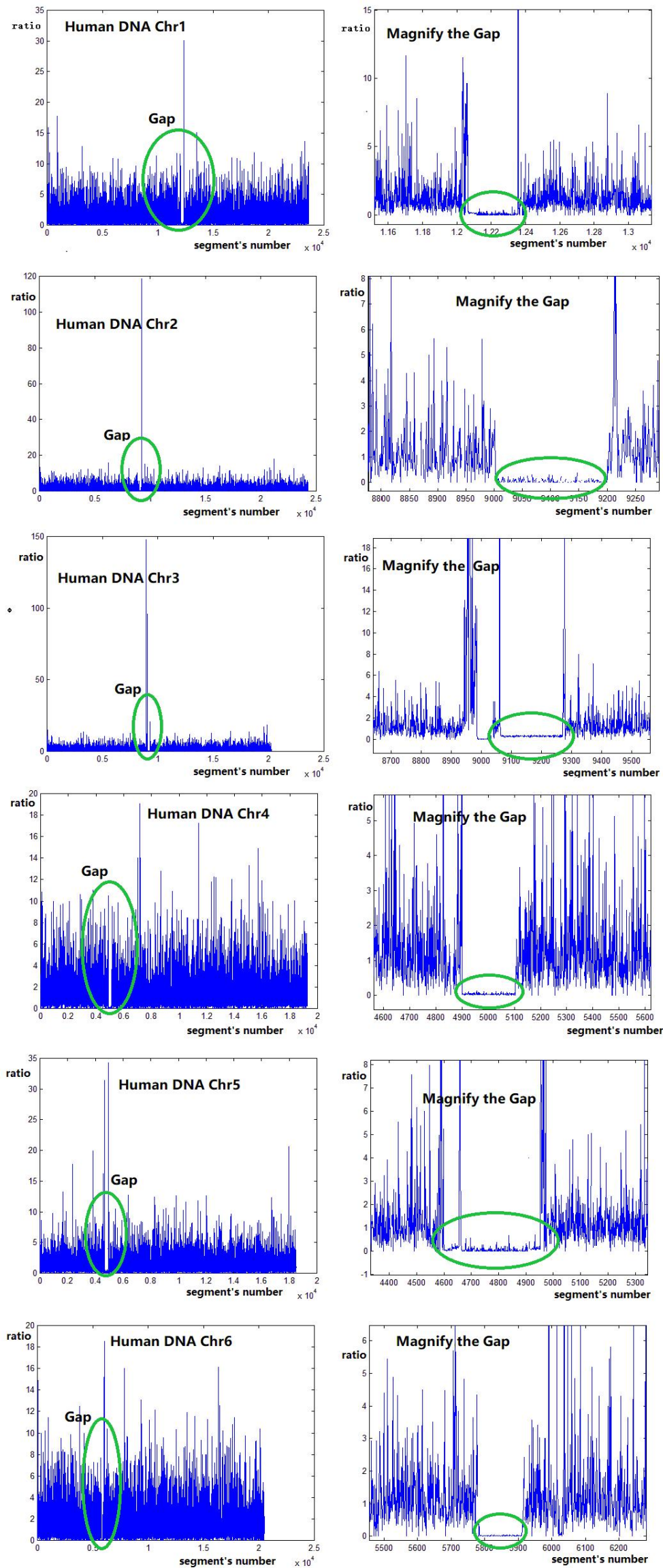

Figure 3. human chromosome. 
In figure 3 It is surprised that human chromosome map has a Gap, magnify the map, we can see more details about the Gap:

1, Every Chromosome has one Gap, the gap has obvious boundaries, and is easy to identify

2, In the Gap, every segment's ratio is less than 0.5 . Outside the Gap, the average ratio is more than 1.0

3, Every chromosome , the length of the Gap is about $1 \%$ the whole chromosome length
4, Chromosome 1 6, every chromosome has two sequences, example to human chromosome1, there are sequence hs_ref_GRCh38.p12_chr1.fa and sequence hs_ref_GRCh38.p12_chr1.gbk, they have almost the same maps : in their maps, the same Gap position and the same Gap length

5, In figure 2, danio rerio, pongo, dog and rat have not the same Gap here

We check all other human chromosomes, from $\mathrm{Chr} 7$ to $\mathrm{Chr} 22$, and $\mathrm{ChrX}$ and $\mathrm{ChrY}$ 

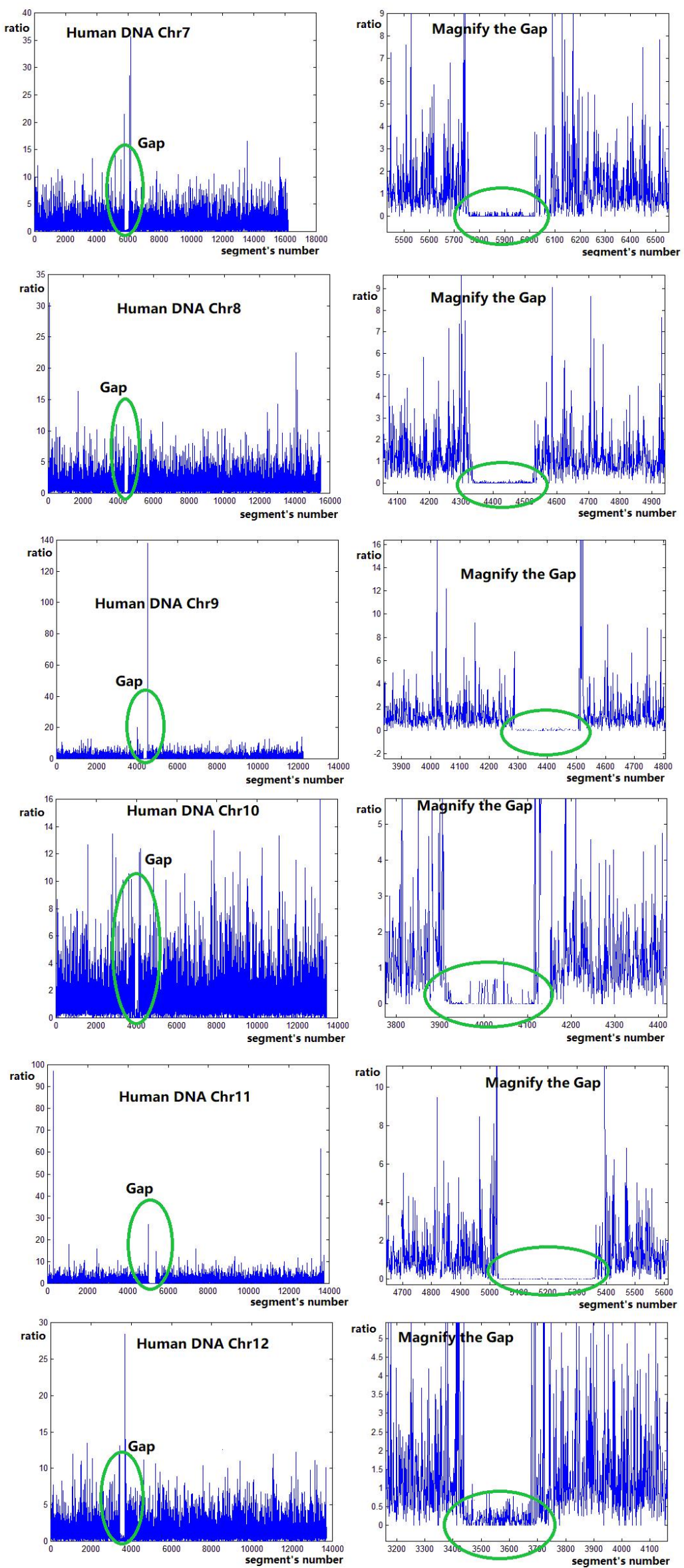

Figure 4. human chromosome. 

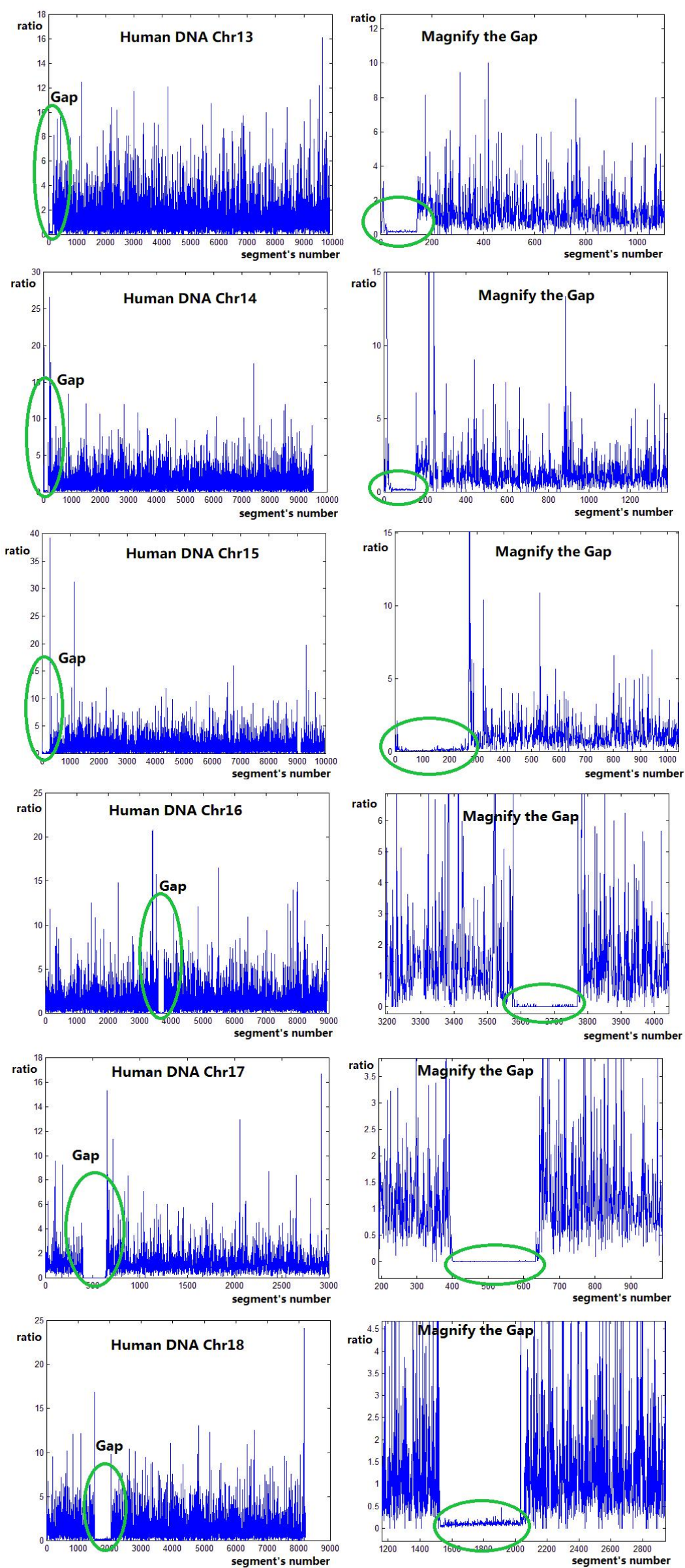

Figure 5. human chromosome. 

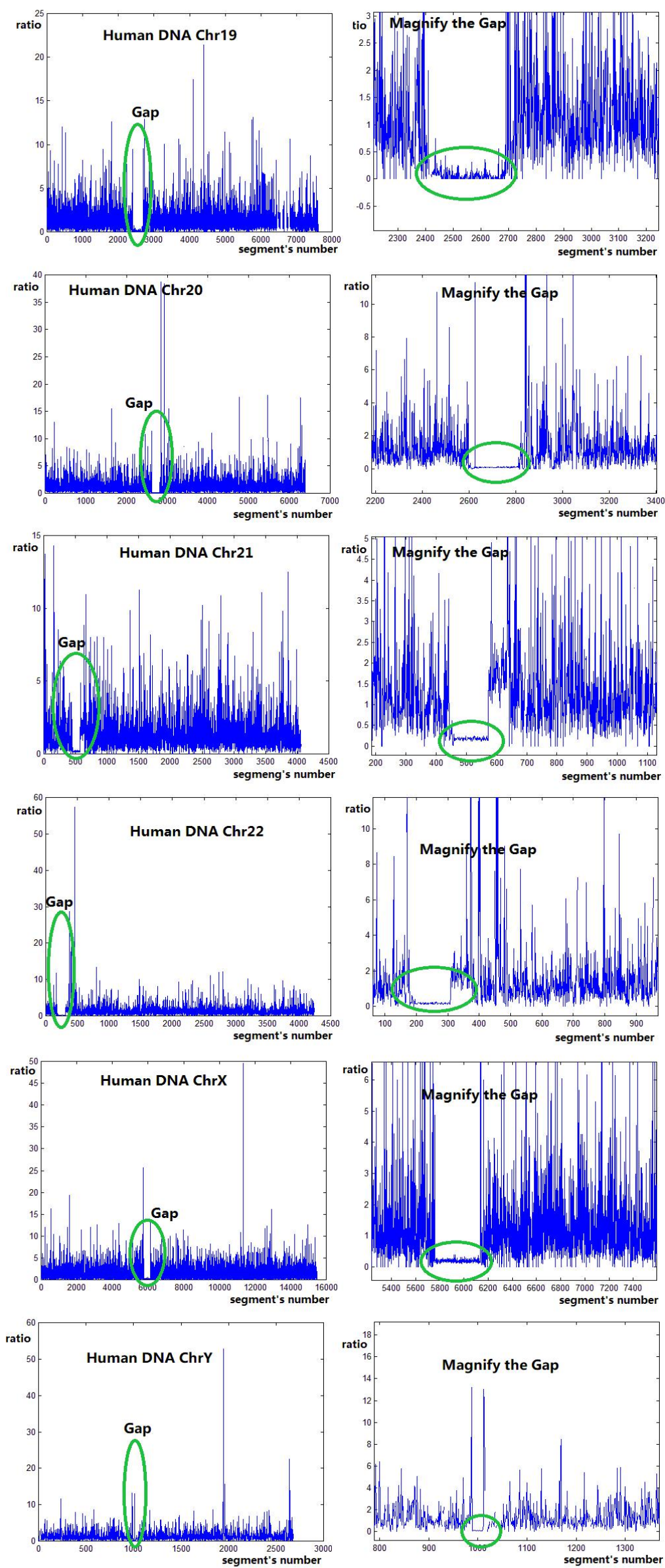

Figure 6. human chromosome. 
We find:

1,To human, every chromosome has one Gap in the map, and only has one Gap.

2,Inside the Gap, the average ratio is less than 0.3 . Inside the Gap, the max ratio in the Gap area is less than 0.5. Out the Gap, the average ratio is more than 1.0

3 , Other creatures include primate, their chromosomes' maps, in figure 2, we can not find any same Gap,It is so interesting!

We are very surprised:

Compared with other biological chromosomes, We collect all biological genome resources in the NCBI, we collected 117 kinds of all other creatures chromosome sequence, a total of about 3500 DNA samples, do the same test, the follow are the samples names:

Ananas_comosus,Anolis_carolinensis,Apis_mellifera ,A rachis_duranensis, Arachis_ipaensis, Asparagus_officinalis , Astyanax_mexicanus ,Beta_vulgaris_subsp._vulgaris,Biomp halaria_glabrata,Ciona_intestinalis ,Capsicum_annuum ,Bo mbus_terrestris ,Canis_familiaris,Carica_papaya ,Callithrix jacchus,Capra_hircus ,Canis_lupus_familiaris,Camelina_sa tiva,Cajanus_cajan,Brassica_rapa,Brassica_oleracea ,Brassic a_napus ,Brachypodium_distachyon ,Bos_taurus ,Bos_indic us ,Cyprinus_carpio ,Cynoglossus_semilaevis ,Cyanistes_ca eruleus ,Cucurbita_pepo_subsp._pepo,Cucumis_sativus ,Cra ssostrea_virginica,Coturnix_japonica, Citrus_sinensis, Cicer arietinum ,Chrysemys_picta ,Chlorocebus_sabaeus ,Drosop hila_busckii ,Drosophila_arizonae ,Daucus_carota_subsp._s ativus ,Danio_rerio ,D_rerio,Ziziphus_jujuba ,Takifugu_rubr ipes,Gossypium_arboreum,Fragaria_vesca,Felis_catus,Dros ophila_navojoa ,Drosophila_miranda ,Ictalurus_punctatus, $\mathrm{H}$ elianthus_annuus,Gossypium_raimondii ,Gossypium_hirsut um ,Gorilla_gorilla ,Glycine_max,Gallus gallus ,Ficedula_a lbicollis,Esox_lucius, Equus_caballus, Elaeis_guineensis, L episosteus_oculatus,Lupinus_angustifolius, Macaca_fascicu laris ,Macaca_mulatta ,Malus_domestica ,Manihot_esculent a ,Meleagris_gallopavo,Microcebus_murinus,Microtus_och rogaster ,Monodelphis_domestica,Mus_caroli ,Mus_muscul us ,Mus_pahari,Musa_acuminata, Ovis_aries, Oryzias_latipe s,Oryza_sativa_Japonica_Group ,Oryza_brachyantha ,Oryct olagus_cuniculus, Ornithorhynchus_anatinus, Oreochromis niloticus, Oncorhynchus_tshawytscha, Oncorhynchus_mykis $\mathrm{s}$,Oncorhynchus_kisutch ,Olea_europaea_var._sylvestris , N umida_meleagris ,Nothobranchius_furzeri ,Nomascus_leuco genys ,Nicotiana_attenuata ,Nasonia_vitripennis,Prunus_per sica,Prunus_mume,Populus_trichocarpa,Pongo_abelii ,Poec ilia_reticulata ,Physcomitrella_patens ,Parus_major,Papio_a nubis ,Taeniopygia_guttata, Sus_scrofa,Spodoptera_litura ,S orghum_bicolor,Solanum_pennellii,Solanum_lycopersicum , Setaria_italica ,Sesamum_indicum ,Sarcophilus_harrisii,Sal velinus_alpinus ,Salmo_salar ,Rosa_chinensis ,R_norvegicu $\mathrm{s}$,Zea_mays,Xiphophorus_maculatus ,Xenopus_tropicalis , Xenopus_laevis,Vitis_vinifera,Vigna_radiata ,Vigna_angular is ,Tribolium_castaneum ,Theobroma_cacao 

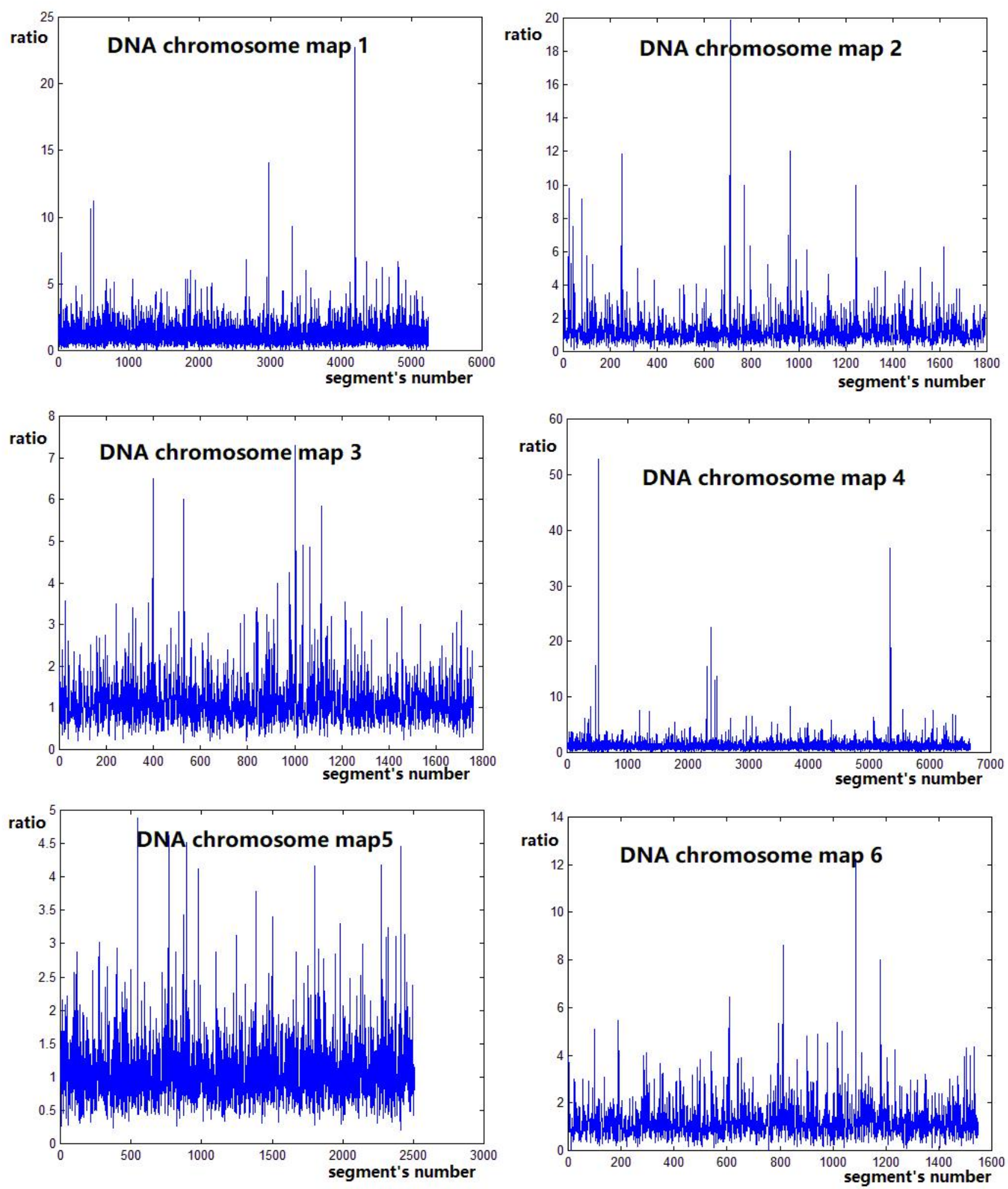

Figure 7. part of other creatures chromosomes maps.

Based on these tests

1,Among other creatures, none of the samples appears the same Gap features in human chromosome image

2,Here test 117 kinds of all other creatures information, include primate creatures, about 3500 chromosome sequences in total

We speculate that the unique characteristics in human chromosomes are the characteristics that other organisms do not possess. 


\section{Discussion}

The human chromosome has an unknown special structure, which is present in every human chromosome and is expressed only once. This structure is also not present in all other organisms the chromosomes.

\section{What is it?}

Do sequencing technologies have errors or mistakes? Does this unknown mistake cause the Gap in the map?

According to figure3, figure4, figure5, figure6, If sequencing errors can cause unknown special structure, we study 117 all kinds of other creatures chromosomes in NCBI, these creatures have $10 \sim 60$ chromosome sequences, assuming that one of the creatures also uses the same error sequencing process like human chromosome sequencing , so all the chromosomes of this creature will appear the same unknown special structure like human chromosomes, in these 3500 DNA samples, there should be at least 10 samples have the same unknown special structure, but the conclusion is that: in the 3500 samples, no one has the special structure like human chromosomes.

Is there such a possibility? there are some kinds of sequencing technology, just one method is wrong. And only human one creature has adopted the only wrong sequencing method, the other 117 species all adopt the other right methods. no no no, it is impossible.

One human chromosome has only one centromere , here we find, every chromosome has only one unique structure, so we compare this unique structure and centromere in follow table 1

Table 1 the structure and the centromere in human chromosome

\begin{tabular}{|c|c|c|c|c|c|c|}
\hline $\begin{array}{c}\text { Human } \\
\text { Chromosome } \\
\text { name }\end{array}$ & File name & $\begin{array}{c}\text { Chromosome Size } \\
\text { (Mbp) }\end{array}$ & $\begin{array}{l}\text { Gap length } \\
\text { (Mbp) }\end{array}$ & $\begin{array}{l}\text { Gap position } \\
\text { (Mbp) }\end{array}$ & $\begin{array}{c}\text { Centromere } \\
\text { position } \\
\text { (Mbp) }\end{array}$ & Centromere kinds \\
\hline Chr1 & hs_ref_GRCh38.p12_chr1.fa & 247.2 & 2.8 & $117.9 \sim 120.6$ & 125 & metacentric \\
\hline Chr2 & hs_ref_GRCh38.p12_chr2.fa & 242.8 & 1.9 & $87.9 \sim 89.8$ & 93.3 & submetacentric \\
\hline Chr3 & hs_ref_GRCh38.p12_chr3.fa & 199.4 & 2.8 & $87.7 \sim 90.5$ & 91 & metacentric \\
\hline Chr4 & hs_ref_GRCh38.p12_chr4.fa & 191.3 & 2 & $47.9 \sim 49.9$ & 50.4 & submetacentric \\
\hline Chr5 & hs_ref_GRCh38.p12_chr5.fa & 180.8 & 3.4 & $44.9 \sim 48.3$ & 48.4 & submetacentric \\
\hline Chr6 & hs_ref_GRCh38.p12_chr6.fa & 170.9 & 1.4 & $56.4 \sim 57.7$ & 61 & submetacentric \\
\hline Chr7 & hs_ref_GRCh38.p12_chr7.fa & 158.8 & 2.6 & $56.2 \sim 58.7$ & 59.9 & submetacentric \\
\hline Chr8 & hs_ref_GRCh38.p12_chr8.fa & 146.3 & 1.9 & $42.3 \sim 44.2$ & 45.6 & submetacentric \\
\hline Chr9 & hs_ref_GRCh38.p12_chr9.fa & 140.4 & 2.7 & $52.3 \sim 55.0$ & 49 & submetacentric \\
\hline Chr10 & hs_ref_GRCh38.p12_chr10.fa & 135.4 & 2 & $38.2 \sim 40.2$ & 40.2 & submetacentric \\
\hline Chr11 & hs_ref_GRCh38.p12_chr11.fa & 134.5 & 3.3 & $49.1 \sim 52.4$ & 53.7 & submetacentric \\
\hline Chr12 & hs_ref_GRCh38.p12_chr12.fa & 132.3 & 2.3 & $33.6 \sim 35.9$ & 35.8 & submetacentric \\
\hline Chr13 & hs_ref_GRCh38.p12_chr13.fa & 114.1 & 1.25 & $0.1 \sim 1.4$ & 17.9 & acrocentric \\
\hline Chr14 & hs_ref_GRCh38.p12_chr14.fa & 106.3 & 1.25 & $0.2 \sim 1.5$ & 17.6 & acrocentric \\
\hline Chr15 & hs_ref_GRCh38.p12_chr15.fa & 100.3 & 2.5 & $0.1 \sim 2.6$ & 19 & acrocentric \\
\hline Chr16 & hs_ref_GRCh38.p12_chr16.fa & 88.8 & 1.9 & $34.9 \sim 36.8$ & 36.6 & metacentric \\
\hline Chr17 & hs_ref_GRCh38.p12_chr17.fa & 78.7 & 3.8 & $21.8 \sim 25.6$ & 24 & submetacentric \\
\hline Chr18 & hs_ref_GRCh38.p12_chr18.fa & 76.1 & 5 & $14.9 \sim 19.7$ & 17.2 & submetacentric \\
\hline Chr19 & hs_ref_GRCh38.p12_chr19.fa & 63.8 & 2.7 & $23.5 \sim 26.3$ & 26.5 & metacentric \\
\hline Chr20 & hs_ref_GRCh38.p12_chr20.fa & 62.4 & 1.9 & $25.6 \sim 27.5$ & 27.5 & metacentric \\
\hline Chr21 & hs_ref_GRCh38.p12_chr21.fa & 46.9 & 1.25 & $4.3 \sim 5.6$ & 13.2 & acrocentric \\
\hline Chr22 & hs_ref_GRCh38.p12_chr22.fa & 49.5 & 1.25 & $1.7 \sim 3.0$ & 14.7 & acrocentric \\
\hline ChrX & hs_ref_GRCh38.p12_chrX.fa & 154.9 & 3.7 & $56.2 \sim 59.9$ & 60.6 & submetacentric \\
\hline ChrY & hs_ref_GRCh38.p12_chrY.fa & 26.1 & 0.2 & $9.7 \sim 9.9$ & 12.5 & acrocentric \\
\hline
\end{tabular}

In human chromosome, unknown special structure is in close contact with centromere

We think :

1,the unknown special structure is an unknown natural structure, is not the error of sequencing technology

2,At present, it exists only in the human chromosome and is related to the centromere. 


\section{References}

[1] Robert Krulwich (2001-04-17). Cracking the Code of Life (Television Show). PBS.

[2] "Economic Impact of the Human Genome Project - Battelle" (PDF). Retrieved 1 August 2013.

[3] https://www.genome.gov/11006943/human-genome-projectcompletion-frequently-asked-questions/. Missing or empty |title $=($ help $)$

[4] "Human Genome Project Completion: Frequently Asked Questions". genome.gov.

[5] "Introducing 'dark DNA' - the phenomenon that could change how we think about evolution".

[6] Olsvik O, Wahlberg J, Petterson B, Uhlén M, Popovic T, Wachsmuth IK, Fields PI (January 1993). "Use of automated sequencing of polymerase chain reaction-generated amplicons to identify three types of cholera toxin subunit B in Vibrio cholerae O1 strains". J. Clin. Microbiol. 31 (1): 22-25. PMC 262614 . PMID 7678018.

[7] Pettersson E, Lundeberg J, Ahmadian A (February 2009). "Generations of sequencing technologies". Genomics. 93 (2): 105-11. doi:10.1016/j.ygeno.2008.10.003. PMID 18992322.

[8] Sanger F, Air GM, Barrell BG, Brown NL, Coulson AR, Fiddes CA, Hutchison CA, Slocombe PM, Smith M (February 1977). "Nucleotide sequence of bacteriophage phi X174 DNA". $\quad$ Nature. 265 (5596): 687-95. Bibcode:1977Natur.265..687S. doi:10.1038/265687a0. PMID 870828 .

[9] "The Next Frontier: Human Viruses" , whatisbiotechnology.org, Retrieved May 3, 2017

[10] Beck S, Pohl FM (1984). "DNA sequencing with direct blotting electrophoresis". EMBO J. 3 (12): 2905-09. PMC 557787 . PMID 6396083.

[11] United States Patent 4,631,122 (1986)

[12] Feldmann H, et al. (1994). "Complete DNA sequence of yeast chromosome II". EMBO J. 13 (24): 5795-809. PMC 395553 . PMID 7813418.

[13] Smith LM, Sanders JZ, Kaiser RJ, Hughes P, Dodd C, Connell CR, Heiner C, Kent SB, Hood LE (12 June 1986). "Fluorescence Detection in Automated DNA Sequence Analysis". $\quad$ Nature. 321 (6071): 674-79. Bibcode:1986Natur.321..674S. doi:10.1038/321674a0. PMID 3713851 .

[14] Prober JM, Trainor GL, Dam RJ, Hobbs FW, Robertson CW, Zagursky RJ, Cocuzza AJ, Jensen MA, Baumeister K (16 Oct 1987). "A system for rapid DNA sequencing with fluorescent chain-terminating dideoxynucleotides". Science. 238 (4825): 336-41. Bibcode:1987Sci...238..336P. doi:10.1126/science.2443975. PMID 2443975.

[15] Adams MD, Kelley JM, Gocayne JD, Dubnick M, Polymeropoulos MH, Xiao H, Merril CR, Wu A, Olde B, Moreno RF (June 1991). "Complementary DNA sequencing: expressed sequence tags and human genome project". Science. 252 (5013): 1651-56. Bibcode:1991Sci...252.1651A. doi:10.1126/science.2047873. PMID 2047873.

[16] Fleischmann RD, Adams MD, White O, Clayton RA, Kirkness EF, Kerlavage AR, Bult CJ, Tomb JF, Dougherty BA, Merrick JM (July 1995). "Whole-genome random sequencing and assembly of Haemophilus influenzae Rd". Science. 269 (5223): 496-512. Bibcode:1995Sci...269..496F. doi:10.1126/science.7542800. PMID 7542800.

[17] Lander ES, Linton LM, Birren B, Nusbaum C, Zody MC, et al. (February 2001). "Initial sequencing and analysis of the human genome". Nature. 409 (6822): 860-921. Bibcode:2001Natur.409..860L. doi:10.1038/35057062. PMID 11237011.

[18] Venter JC, Adams MD, et al. (February 2001). "The sequence of the human genome". Science. 291 (5507): 1304-51. Bibcode:2001Sci...291.1304V. doi:10.1126/science.1058040. PMID 11181995.

[19] Maxam AM, Gilbert W (February 1977). "A new method for sequencing DNA". Proc. Natl. Acad. Sci. USA. 74 (2): 56064. Bibcode:1977PNAS...74..560M. doi:10.1073/pnas.74.2.560. PMC 392330 . PMID 265521.

[20] Sanger F, Nicklen S, Coulson AR (December 1977). "DNA sequencing with chain-terminating inhibitors". Proc. Natl. Acad. Sci. USA. 74 (12): 5463-77. Bibcode:1977PNAS...74.5463S. doi:10.1073/pnas.74.12.5463. PMC 431765 . PMID 271968.

[21] Sanger F, Coulson AR (May 1975). "A rapid method for determining sequences in DNA by primed synthesis with DNA polymerase". J. Mol. Biol. 94 (3): 441-48. doi:10.1016/0022-2836(75)90213-2. PMID 1100841.

[22] Wetterstrand, Kris. "DNA Sequencing Costs: Data from the NHGRI Genome Sequencing Program (GSP)". National Human Genome Research Institute. Retrieved 30 May 2013.

[23] Kawashima, Eric H.; Laurent Farinelli; Pascal Mayer (200505-12). "Patent: Method of nucleic acid amplification". Retrieved 2012-12-22.

[24] Staden R (11 Jun 1979). "A strategy of DNA sequencing employing computer programs". Nucleic Acids Research. 6 (7): 2601-10. doi:10.1093/nar/6.7.2601. PMC 327874 . PMID 461197.

[25] P. Mayer,L. Farinelli, G. Matton, C. Adessi, G. Turcatti, J. J. Mermod, E. Kawashima.DNA colony massively parallel sequencing ams 98 presentation

[26] U.S. Patent 5,641,658

[27] Braslavsky I, Hebert B, Kartalov E, Quake SR (April 2003). "Sequence information can be obtained from single DNA molecules". Proc. Natl. Acad. Sci. USA. 100 (7): 3960-64. Bibcode:2003PNAS..100.3960B.

doi:10.1073/pnas.0230489100. PMC 153030 . PMID 12651960 .

[28] Alberts, Bruce; Bray, Dennis; Hopkin, Karen; Johnson, Alexander; Lewis, Julian; Raff, Martin; Roberts, Keith; Walter, Peter (2014). Essential Cell Biology (4 ed.). New York, NY: Garland Science. p. 183. ISBN 978-0-8153-4454-4.

[29] Pollard, T.D. (2007). Cell Biology. Philadelphia: Saunders. pp. 200-203. ISBN 978-1-4160-2255-8.

[30] Pollard, TD (2007). Cell Biology. Philadelphia: Saunders. pp. 227-230. ISBN 978-1-4160-2255-8.

[31] Pluta, A.; A.M. Mackay; A.M. Ainsztein; I.G. Goldberg; W.C. Earnshaw (1995). "The centromere: Hub of chromosomal activities". Science. $270 \quad$ (5242): 1591-1594. doi:10.1126/science.270.5242.1591. PMID 7502067.

[32] "Sister chromatid cohesion". Genetics Home Reference. United States National Library of Medicine. May 15, 2011. 